\title{
The cost of getting personal
}

\author{
The recent report of an antisense oligonucleotide therapy designed for only one patient spotlights \\ regulatory, economic and ethical issues that must be grappled with in an era of accelerated development \\ of 'ultra-personalized' drugs.
}

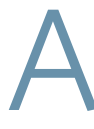

report published in October described the case of a young girl, Mila, with Batten's disease, a progressive fatal neurodegenerative disorder. After discovering mutations in each of the two copies of the CLN7 gene in Mila's genome, researchers were able to design an antisense oligonucleotide, termed milasen, that could restore the correct splicing of one of the mutant alleles. The time that elapsed from diagnosis to dosing-including identification of the antisense oligonucleotide that could correct splicing in cells derived from the patient and toxicological tests in rodents-was less than a year, a remarkable feat. Over the course of 300 days after initiation of drug administration, via repeated intrathecal injections, treatment seemed to be safe. While some aspects of disease progression appeared to continue unabated, the frequency and duration of seizures decreased, suggesting a beneficial effect of the drug.

The development of milasen follows in the footsteps of splice-modulating antisense oligonucleotides previously approved for treating other types of genetic disease, including nusinaren for spinal muscle atrophy and eteplirsen for Duchenne muscular dystrophy. However, in the case of milasen, only one patient in the world is known to have the specific type of mutation that can be treated with the antisense oligonucleotide, making it a true ' $n$-of-one' drug. News reports indicate that individualized antisense oligonucleotides are in the works for patients with other rare genetic diseases, including a patient with amyotrophic lateral sclerosis who has already been treated with such a drug.

Mila's story is undoubtedly compelling in describing how cutting-edge medicine can be deployed with great speed for the treatment of a young girl whose condition was rapidly worsening. What is not yet clear is whether this case is a template that can be routinely reproduced for the development of $n$-of-one therapies. Among the issues that regulators must address are determining how much animal toxicity testing and preclinical evidence of efficacy are needed before giving the go-ahead for dosing of a patient with a rapidly progressive disease. Beyond these practical questions, there are broader economic and ethical issues. For the development of milasen, Mila's family managed to raise $\$ 3$ million through crowdsourcing and succeeded in enlisting academic researchers to take up the challenge. Such resources, in terms of both money and expertise, are not easily replicated.

Optimistically, it may be feasible to scale up production and testing of antisense oligonucleotides, increasing the speed with which they can be developed and lowering their cost. For example, antisense oligonucleotides targeting different genetic defects but made with the same types of chemical modifications to their DNA backbone may have similar toxicological, pharmacokinetic and biodistribution properties. The patient population that could be targeted by this type of approach is larger than might be guessed. Rare single-gene disorders affect about $1 \%$ of the population, of which it has been estimated that $15 \%$ could be targeted by individualized antisense oligonucleotides.

Looming over this type of ultrapersonalized therapy-as well as others, such as chimeric antigen receptor (CAR) $\mathrm{T}$ cell therapy for cancer-is the issue of how society will manage to afford them. When treatment with a CAR T cell therapy costs $\$ 475,000$, new types of payment models seem to be needed. As Fischer et al. discuss in this issue in the context of gene therapy, one potential option is a valuebased approach for payment, in which the price of a drug is more directly tied to the value it brings to the patient, an approach that has been pushed forward in both the United States and Europe. Along these lines, in Germany a 'pay-for-performance' agreement was recently implemented for Kymriah (tisagenlecleucel), a CAR T cell therapy, according to which Novartis will pay back part of the price of the drug to a health insurance fund if patient survival doesn't reach an agreed-upon threshold.

These enormously expensive types of therapy may also exacerbate inequities in access to medicine-would access be restricted only to those in the highest socioeconomic stratum? The advances of precision medicine must be available to all sectors of society, not just for the most privileged. In a step in that direction, a joint initiative of the Bill \& Melinda Gates Foundation and the US National Institutes of Health, announced in October, aims to develop new genetic therapies for HIV and sickle cell disease that can be used globally in low-resource, high-disease-burden regions, such as sub-Saharan Africa. The initiative plans to design gene-based cures that can be administered in areas with limited medical infrastructure.

As we head into the next decade, the development of personalized therapies will only accelerate, but they come with a hefty price tag. Drug developers and payers need to devise innovative solutions for balancing the need to fund future research investment with the accessibility of these therapies. Such personalized approaches also need to be balanced with public health initiatives that reach large swaths of the population. Amid the excitement of the possibility of high-tech cures, the goal of improving the health of all must not be forgotten.

Published online: 5 December 2019 https://doi.org/10.1038/s41591-019-0700-3 